\title{
Pretreatment systemic inflammation response index (SIRI) is an independent predictor of survival in unresectable stage III non- small cell lung cancer treated with chemoradiotherapy: a two- center retrospective study
}

\author{
Min $\mathrm{Hu}^{1 \#}$, Qinghua Xu${ }^{1 \#}$, Shuangyan Yang ${ }^{1 \#}$, Shuiyun $\mathrm{Han}^{2}$, Yaoyao Zhu', Qingren Lin ${ }^{2}$, Xiaojiang Sun ${ }^{2}$, \\ Hui Liu', Yaping $\mathrm{Xu}^{1}$ \\ ${ }^{1}$ Department of Radiotherapy, Shanghai Pulmonary Hospital, Tongji University School of Medicine, Shanghai, China; ${ }^{2}$ Department of Thoracic \\ Radiotherapy, Cancer Hospital of the University of Chinese Academy of Sciences (Zhejiang Cancer Hospital), Hangzhou, China \\ Contributions: (I) Conception and design: H Liu, Y Xu; (II) Administrative support: Y Xu; (III) Provision of study materials or patients: Y Zhu, Q Lin, \\ X Sun; (IV) Collection and assembly of data: S Han, S Yang; (V) Data analysis and interpretation: M Hu, Q Xu; (VI) Manuscript writing: All authors; \\ (VII) Final approval of manuscript: All authors. \\ \#These authors contributed equally to this work. \\ Correspondence to: Yaping Xu. Department of Radiotherapy, Shanghai Pulmonary Hospital, Tongii University School of Medicine, No. 507, Zhengmin \\ Road, Yangpu District, Shanghai 200433, China. Email: xuyaping1207@163.com; Hui Liu. Department of Radiotherapy, Shanghai Pulmonary \\ Hospital, Tongji University School of Medicine, No. 507, Zhengmin Road, Yangpu District, Shanghai 200433, China. Email: huiliu1127@163.com.
}

Background: Circulating immune cells influence the efficacy of cancer therapy. This study aimed to investigate the prognostic values of different peripheral blood leukocyte (PBL) biomarkers in non-small lung cancer (NSCLC) patients treated with chemoradiotherapy.

Methods: An independent cohort of 176 stage III NSCLC patients who were diagnosed at Shanghai Pulmonary Hospital and Zhejiang Cancer Hospital between April, 2010, and September, 2018, and had available pretreatment peripheral blood tests was enrolled. The patients were all treated with concurrent or sequential chemoradiotherapy according to international clinical guidelines, with conventional fractionated radical radiotherapy. The receiver operating characteristic curve and the Youden index were used to determine the optional cutoff values of PBL biomarkers for distinguishing prognosis. Univariate and multivariate Cox proportional hazards regression analyses were performed to identify the factors significantly correlated with overall survival.

Results: The cohort had a median follow-up time of 21.7 (3.1-121) months. The 3- and 5-year OS rates of all patients were $34.7 \%$ and $27.5 \%$, respectively. Univariate analysis showed that gender $(\mathrm{P}=0.011)$, smoking $(\mathrm{P}=0.011)$, tumor-node-metastasis $(\mathrm{TNM})$ stage $(\mathrm{P}=0.002)$, pretreatment peripheral blood neutrophil-toleukocyte ratio $(\mathrm{P}=0.013)$, and systemic inflammation response index (SIRI, $\mathrm{P}<0.001)$ were all correlated with OS in NSCLC patients. Moreover, multivariate analysis revealed that TNM stage (HR $=1.541,95 \%$ CI: $1.166-2.036, \mathrm{P}=0.010)$ and SIRI $(\mathrm{HR}=1.868,95 \% \mathrm{CI}: 1.016-3.436, \mathrm{P}=0.018$ ) were significantly and independently associated with OS. However, the median OS of stage IIIB NSCLC patients with low

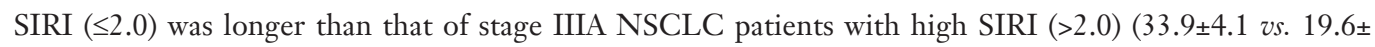
2.5 months).

Conclusions: Pretreatment peripheral blood SIRI was found to be a simple independent predictor of OS in stage III NSCLC patients who underwent chemoradiotherapy. As a novel prognostic marker, the prognostic value of the SIRI is superior to that of the NLR. Low SIRI could be a better prognostic stratification factor for NSCLC patients with different TNM stages.

Keywords: Non-small cell lung cancer (NSCLC); prognosis; biomarkers; systemic inflammation response index (SIRI); neutrophil-to-lymphocyte ratio (NLR) 
Submitted Aug 11, 2020. Accepted for publication Oct 16, 2020.

doi: 10.21037/atm-20-6484

View this article at: http://dx.doi.org/10.21037/atm-20-6484

\section{Introduction}

Non-small cell lung cancer (NSCLC) is one of the most prevalent cancers globally and comprises at least $85 \%$ of all new lung cancer cases (1). Approximately $30 \%$ of NSCLC patients are locally advanced (stage III) at the time of diagnosis, and many of these patients cannot achieve complete resection due to the tumor size or location, or patient-related factors (2). Large or invasive T4 tumors, N3 lymph node involvement, poor pulmonary function of patients, and cardiovascular comorbidities are all common reasons for unresectability $(3,4)$. For patients with unresectable stage III NSCLC, chemotherapy combined with radiotherapy remains the standard treatment, while consolidation immunotherapy after concurrent chemoradiotherapy has been emerging as a treatment selection (5).

The prognosis of NSCLC is generally evaluated preliminarily according to the tumor-node-metastasis (TNM) staging system, histology, and oncogenic mutations, which guide patient management strategies (6). However, the clinical prognosis can vary, even for patients in the same category (6). Therefore, to optimize risk stratification and to predict prognosis more accurately, as well as to further inform individualized treatment management decisions, this population of NSCLC patients requires additional effective prognostic biomarkers.

Inflammatory reaction and immune surveillance are considered to be pivotal hallmarks relevant to cancer development and outcomes of cancer therapy (7-9). Because peripheral blood immune cells directly reflect the condition of the immune system, peripheral blood leukocyte (PBL) biomarkers such as the absolute counts of neutrophils, monocytes, and lymphocytes, along with the neutrophil-tolymphocyte ratio (NLR), have been reported to be effective predictors of prognosis in different tumor models. High lymphocyte and low neutrophil counts have been associated with better prognosis in various tumor models (10-13). Moreover, the novel systemic inflammation response index (SIRI), which integrates different inflammatory cells (neutrophils, monocytes, and lymphocytes), has been proved to be a promising prognostic predictor in different cancers $(14,15)$.
However, few previous studies to date have studied the prognostic value of pretreatment SIRI in unresectable locally advanced NSCLC patients treated with chemoradiotherapy. In this study, we retrospectively analyzed the survival data of patients with stage III NSCLC, and explored the prognostic role and optimal cutoffs of different pretreatment inflammatory biomarkers, including SIRI and NLR. We present the following article in accordance with the REMARK reporting checklist (available at http://dx.doi.org/10.21037/atm-20-6484).

\section{Methods}

\section{Patients}

Unresectable stage III NSCLC patients who underwent chemoradiotherapy at the Department of Radiotherapy, Shanghai Pulmonary Hospital or Zhejiang Cancer Hospital between April, 2010, and September, 2018, were retrospectively enrolled. All tumor staging was assessed according to the American Joint Committee on Cancer (AJCC) guidelines, 8th edition (16). The inclusion criteria were: (I) NSCLC newly diagnosed in one of our institutions; (II) NSCLC pathologically confirmed; (III) stage III disease according to the TNM staging system (5); (IV) treated with both radiotherapy and chemotherapy; and $(\mathrm{V})$ pretreatment peripheral blood tests available. The exclusion criteria were: (I) underwent surgical resection; (II) had a second primary malignancy; (III) clinical evidence of active infection or inflammation; (IV) hematological disease or autoimmune diseases; or $(\mathrm{V})$ insufficient clinical or laboratory data.

A total of 176 patients with unresectable stage III NSCLC who met the inclusion criteria were included in the study. Data on the patients' clinicopathological characteristics including gender, age, smoking status, histopathology, TNM stage, treatment regimen, and pretreatment PBL biomarkers were collected. This retrospective study received approval from the Institutional Review Boards of Shanghai Pulmonary Hospital and Zhejiang Cancer Hospital (approval No.19231ZL). Informed consent was obtained from each patient who participated in the study. All procedures 
performed in this study involving human participants were in accordance with the Declaration of Helsinki (as revised in 2013).

The optimal cutoffs for PBL biomarkers were identified using receiver operating characteristic (ROC) curve analysis, with overall survival (OS) as the outcome of interest. We use the optimal cutoffs of the PBL biomarkers as boundaries to group the cohort into different OS prognosis. The collected pretreatment PBL biomarkers included the absolute counts of neutrophils, monocytes, and lymphocytes, NLR, and SIRI. The NLR and SIRI were calculated as follows: NLR = neutrophils count/lymphocytes count, and SIRI = neutrophils $\times$ monocytes/lymphocytes.

\section{Treatment methods}

Patients had received concurrent or sequential chemoradiotherapy, which included conventional fractionated definitive radiation (between 54 and 66 Gy) with appropriate organs-at-risk (OARs) dose constraints using advanced technologies and more than two cycles of platinum-based chemotherapy based on NCCN guidelines.

\section{Follow-up}

Patients were followed up regularly from the date of diagnosis to April 14, 2020, or the date of death. The endpoint of the study was OS, which was defined as the time from the date of diagnosis to the date of death, or to the last follow-up.

\section{Statistical analysis}

All statistical analyses were performed using SPSS 21.0 statistical software (IBM Corp., Armonk, NY, USA). Survival curves were analyzed by the Kaplan-Meier method and compared by log-rank test. The optional cutoff values of the PBL biomarkers for distinguishing prognosis were determined by calculating the area under the ROC curve (AUC), using the maximum principle of the Youden index. Pearson's chi-square test was used to compare categorical variables. Univariate and multivariate Cox regression proportional hazards models were performed, with hazard ratio (HRs) and $95 \%$ confidence intervals (95\% CI) expressing the intensity of correlations between the observed factors and OS. A two-sided $\mathrm{P}<0.05$ was considered statistically significant.

\section{Results}

\section{Patient characteristics}

The 176 patients in this study had a mean age of $62.9 \pm$ 8.0 (range, 33-80) years; 157 (89.2\%) patients were male and $19(10.8 \%)$ were female. The median followup was 21.7 (range, 3.1-121) months. The OS rate at 3 and 5 years was $34.7 \%$ and $27.5 \%$, respectively. Of the patients, $123(69.9 \%)$ had a smoking history. In terms of histological type, 87 patients $(49.4 \%)$ had adenocarcinoma and 65 patients $(36.9 \%)$ had squamous cell carcinoma, while for 24 patients (13.6\%), the type was unspecified. Concurrent chemoradiotherapy (CCRT) and sequential chemoradiotherapy (SCRT) were received by 32 (18.2\%) and $144(81.8 \%)$ patients, respectively. According to TNM stage, 57 (32.4\%) patients had stage IIIA disease, 97 (55.1\%) had stage IIIB, and $22(12.5 \%)$ had stage IIIC. Table 1 shows the clinicopathological characteristics and levels of inflammatory response markers of the 176 stage III NSCLC patients.

\section{Optimal cut-off value for PBL biomarkers}

Time-dependent ROC curves were used to analyze the predictive effects of the PBL biomarkers and to determine the optional cutoff values of different markers for predicting OS prognosis. The AUCs of SIRI and NLR were 0.714 (95\% CI: 0.604-0.823, P=0.001) and 0.693 (95\% CI: $0.569-0.817, \mathrm{P}=0.002$ ), respectively (Figure 1 ), The AUC for absolute neutrophil count was 0.564 (95\% CI: $0.436-$ 0.692, $\mathrm{P}=0.312$ ); the AUC for absolute monocyte count was 0.568 (95\% CI: $0.434-0.703, \mathrm{P}=0.283$ ); and the AUC for absolute lymphocyte count was 0.508 (95\% CI: 0.388-0.627, $\mathrm{P}=0.906)$. According to the maximum principle of the Youden index, the optimal cutoff values for SIRI and NLR were 2.0 and 2.7, respectively. In the survival analysis, the patients were further grouped according to the optimal cutoff values of pretreatment SIRI and NLR.

\section{Prognostic analysis}

The median follow-up time was 21.7 (range, 3.1121.0) months. The 3 - and 5-year OS rates for all included unresectable stage III NSCLC patients treated with chemoradiotherapy were $34.7 \%$ and $27.5 \%$, respectively.

Survival curves were estimated using the Kaplan-Meier method and compared by log-rank test, and we found that factors correlated with different prognosis in the Kaplan- 
Table 1 Clinicopathological characteristics and levels of inflammatory response markers of 176 patients with stage III NSCLC

\begin{tabular}{|c|c|}
\hline Variable & No. of patients (\%)/median (range) \\
\hline \multicolumn{2}{|l|}{ Age (years) } \\
\hline$\leq 60$ & $68(38.6)$ \\
\hline$>60$ & $108(61.4)$ \\
\hline \multicolumn{2}{|l|}{ Sex } \\
\hline Male & $157(89.2)$ \\
\hline Female & $19(10.8)$ \\
\hline \multicolumn{2}{|l|}{ Smoking status } \\
\hline Ever & $123(69.9)$ \\
\hline Never & $53(30.1)$ \\
\hline \multicolumn{2}{|l|}{ Histological type } \\
\hline LUSC & $65(36.9)$ \\
\hline LUAD & $87(49.4)$ \\
\hline NOS & $24(13.6)$ \\
\hline \multicolumn{2}{|l|}{ TNM stage } \\
\hline IIIA & $57(32.4)$ \\
\hline IIIB & $97(55.1)$ \\
\hline IIIC & $22(12.5)$ \\
\hline \multicolumn{2}{|l|}{ Treatment } \\
\hline CCRT & $32(18.2)$ \\
\hline SCRT & $144(81.8)$ \\
\hline Neutrophils, $\times 10^{9} / \mathrm{L}$ & $4.65(0.10-18.67)$ \\
\hline Monocytes, $\times 10^{9} / \mathrm{L}$ & $0.49(0-2.01)$ \\
\hline Lymphocytes, $\times 10^{9} / \mathrm{L}$ & $1.74(0.28-4.06)$ \\
\hline NLR & $2.80(0.10-35.20)$ \\
\hline SIRI & $1.35(0-25.00)$ \\
\hline
\end{tabular}

*, $\mathrm{P}<0.05$ is considered significant. NSCLC, non-small cell lung cancer; LUSC, lung squamous carcinoma; LUAD, lung adenocarcinoma; NOS, not otherwise specified; TNM, tumor node metastasis; CCRT, concurrent chemoradiotherapy; SCRT, Sequential chemoradiotherapy; NLR, neutrophil-to-lymphocyte ratio; SIRI, systemic inflammation response index.

Meier survival analysis were sex $(\mathrm{P}=0.008)$, smoking status $(\mathrm{P}=0.010)$, NLR $(\mathrm{P}=0.012)$, and SIRI $(\mathrm{P}<0.001)$. In particular, among the different $\mathrm{PBL}$ biomarkers, differences prognosis between NSCLC patients in high and low NLR groups, SIRI groups were illustrated (Figure 2).

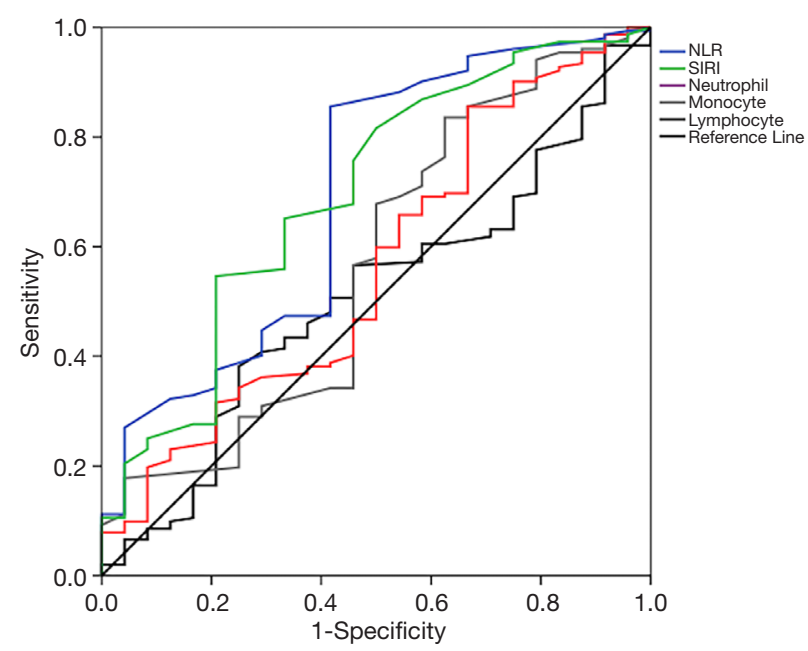

Figure 1 Receiver operating characteristic curve analysis of the optimal cutoff value of absolute neutrophil count, absolute monocyte count, absolute lymphocyte count, neutrophil-tolymphocyte ratio (NLR), and systemic inflammation response index (SIRI) in patients with unresectable stage III non-small cell lung cancer. Areas under the curve for overall survival were 0.714 and 0.693 for the SIRI and NLR, respectively $(\mathrm{P}<0.05)$. $\mathrm{P}>0.05$ for all other indicators.

As shown in Table 2, in univariate Cox analysis, the factors significantly correlated with OS were sex (HR: 2.907, 95\% CI: 1.277-6.620, $\mathrm{P}=0.011)$, smoking status (HR: 1.725, 95\% CI: $1.132-2.629, \mathrm{P}=0.011$ ), TNM stage (IIIBHR: $1.727,95 \%$ CI: $1.053-2.824$; IIIC-HR: $2.724,95 \%$ CI: $1.550-4.784 ; \mathrm{P}=0.002$ ), NLR $>2.7$ (HR: $1.590,95 \%$ CI: $1.101-2.295, \mathrm{P}=0.013$ ), and SIRI $>2.0$ (HR: $2.311,95 \%$ CI: 1.472-3.630, $\mathrm{P}<0.001)$. Furthermore, in multivariate analysis using the Cox proportional hazards model, both TNM stage (HR: 1.541, 95\% CI: 1.166-2.036, $\mathrm{P}=0.010$ ) and SIRI (HR: $1.868,95 \% \mathrm{CI}: 1.016-3.436, \mathrm{P}=0.018$ ) were independent prognostic factors for OS.

\section{Pretreatment SIRI as a risk stratification indicator}

In Kaplan-Meier survival analysis, NSCLC patients in different TNM stage III subgroups showed significant differences in OS $(\mathrm{P}=0.001)$ (Figure $3 A)$. Furthermore, value of SIRI on risk stratification in locally-advanced NSCLC patients was explored. As shown in Figure 3B, the median OS of stage IIIA NSCLC patients with low SIRI was not reached, which was superior to that of the high SIRI patients $(23.8 \pm 4.9$ months). Stage IIIB patients 

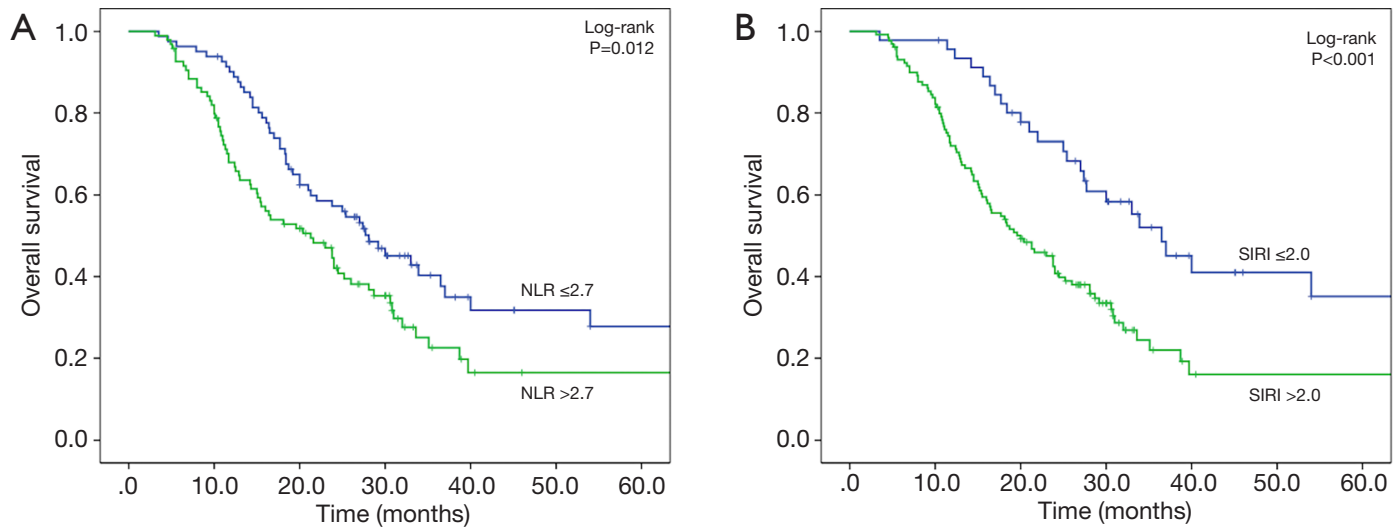

Figure 2 Kaplan-Meier curves for overall survival (OS) according to the neutrophil-to-lymphocyte ratio (NLR) (A) and systemic inflammation response index (SIRI) (B). A high (>2.7) NLR and high (>2.0) SIRI were associated with significantly shorter OS.

Table 2 Univariate and multivariate Cox proportional hazards analyses for overall survival (OS) in the cohort

\begin{tabular}{|c|c|c|c|c|c|}
\hline Variable & Median OS (95\% Cl) & \multicolumn{2}{|c|}{ Univariate analysis } & \multicolumn{2}{|c|}{ Multivariate analysis } \\
\hline \multicolumn{6}{|c|}{ Gender, n (\%) } \\
\hline Male & $23.8(19.6-27.9)$ & 2.907 (1.277-6.620) & $0.011^{*}$ & $1.798(0.723-4.464)$ & 0.150 \\
\hline Female & - & 1 & & 1 & \\
\hline$\leq 60$ years & $24.5(17.9-31.1)$ & 1 & 0.281 & & \\
\hline$>60$ years & $23.8(17.5-30.1)$ & $1.231(0.843-1.801)$ & & & \\
\hline \multicolumn{6}{|c|}{ Histological type } \\
\hline LUSC & $27.0(15.9-38.1)$ & 1 & 0.138 & & \\
\hline Never & $32.0(17.9-46.1)$ & 1 & & 1 & \\
\hline \multicolumn{6}{|c|}{ TNM stage, n (\%) } \\
\hline IIIA & $30.8(15.9-45.7)$ & 1 & $0.002^{*}$ & $1.541(1.166-2.036)$ & $0.010^{*}$ \\
\hline IIIB & $24.0(19.3-28.7)$ & 1.727 (1.054-2.825) & & & \\
\hline IIIC & $16.4(12.1-20.7)$ & 2.725 (1.550-4.785) & & & \\
\hline \multicolumn{6}{|l|}{ NLR } \\
\hline$\leq 2.7$ & $29.2(19.6-38.8)$ & 1 & $0.013^{\star}$ & 1 & 0.836 \\
\hline
\end{tabular}

${ }^{*}, \mathrm{P}<0.05$ is considered significant. LUSC, lung squamous carcinoma; LUAD, lung adenocarcinoma; TNM, tumor node metastasis; NLR, neutrophil-lymphocyte ratio; SIRI, systemic inflammation response index. 

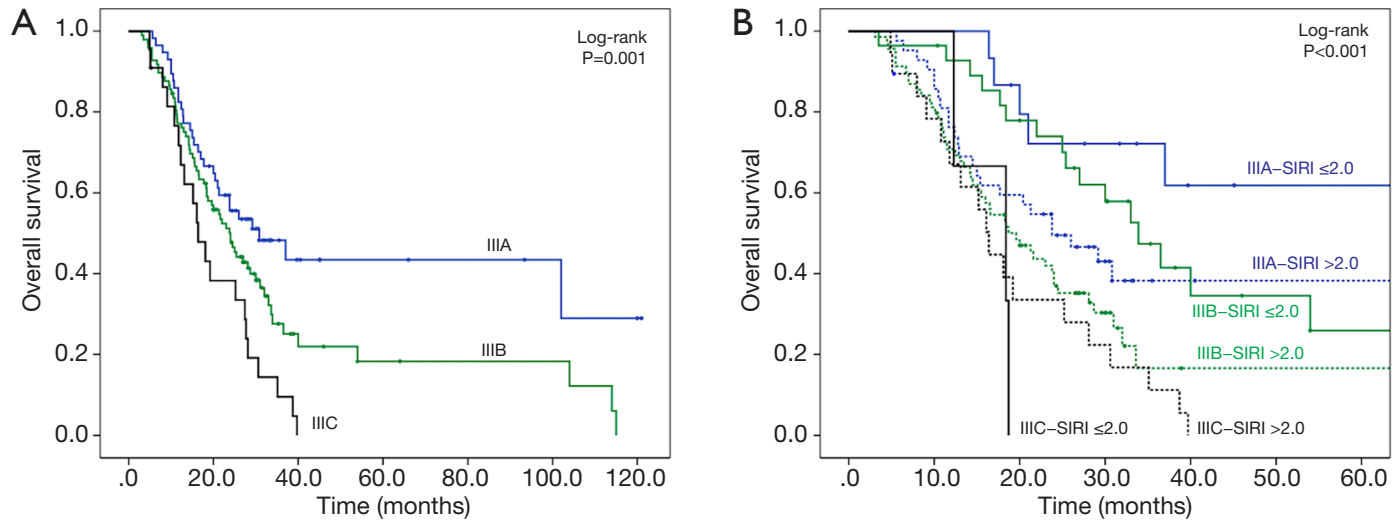

Figure 3 Kaplan-Meier curves showing overall survival in non-small cell lung cancer patients in different tumor node metastasis (TNM) stage III subgroups (A) and survival analysis of the high systemic inflammation response index (SIRI) (>2.0) and low SIRI ( $\leq 2.0)$ groups with different subgroups according to TNM stage III (B).

with low SIRI had a longer median OS $(33.9 \pm 4.1$ months $)$ than stage IIIB patients with high SIRI (19.6 2.5 months). Furthermore, among stage IIIC patients, the low SIRI subgroup had a better median OS than the high SIRI subgroup (18.4 \pm 5.0 vs. $16.4 \pm 1.3$ months). In particular, we found that stage IIIA patients with high SIRI had a worse 3 -year OS rate than stage IIIB patients with low SIRI (9.5\% vs. $32.1 \%, \mathrm{P}=0.007)$.

\section{Discussion}

In this study, the prognostic data of 176 unresectable stage III NSCLC patients treated with concurrent or sequential chemotherapy and conventional fractionated radical radiotherapy in two institutions were retrospectively analyzed. Although TNM staging was certificated as an efficient predictive prognostic factor in our study, SIRI was validated to be a novel and independent factor for predicting OS, outperforming NLR. Furthermore, we validated SIRI and NLR with the respective optimal cutoffs of 2.0 and 2.7 in an independent, multi-institutional cohort of patients. Our study is the first to explore the potential of pretreatment SIRI in predicting survival among patients in the same TNM stage, in particular those with unresectable locally advanced lung cancer patients treated with chemoradiotherapy.

A growing body of evidence indicates that systemic inflammatory biomarkers in peripheral blood can be used to predict treatment outcomes in different solid tumors, included prostate cancer, colorectal cancer, esophageal squamous cell carcinoma, melanoma, and NSCLC
$(7,9,17)$. The potential mechanisms underlying the effects of different inflammatory biomarkers on prognosis are unclear; however, they are possibly related to changes in the tumor immune microenvironment. Moreover, tumor immune microenvironment changes are closely associated with inflammatory and immune cell distribution in the peripheral blood (7). As previously reported, lymphocytes play a crucial role in cancer therapy, and infiltration by lymphocytes stimulates the production of more proinflammatory factors, which promote cytotoxic cell death and inhibit tumor cell proliferation and migration $(17,18)$. On the contrary, neutrophils have been indicated to have tumor-promoting properties. Relative increases in neutrophils in the peripheral blood accompanied by the up-regulation of tumorigenic and angiogenic factors, such as vascular endothelial growth factor (VEGF), nuclear factor kappa-B (NF-kB), C-X-C motif chemokine ligand 8 (CXCL8), granulocyte colony-stimulating factor (G-CSF), and transforming growth factor- $\beta 1$ (TGF- $\beta 1$ ), induce a tumor-promoting environment $(19,20)$. A high number of circulating neutrophils has been reported to predict poor therapeutic effects and worse prognosis of patients treated with chemotherapy, radiotherapy, or checkpoint inhibitors in several cancers $(13,21,22)$. Meanwhile, elevated circulating absolute monocyte count has also been shown to predict worse survival of patients in several tumor models. Monocytes were found to differentiate into tumorassociated macrophages (17), secrete tumor necrosis factor alpha (TNF- $\alpha$ ) and VEGF to facilitate tumor growth and angiogenesis, and inhibit the antitumor immune response in vivo. They have also been shown to promote tumor cell 
migration by secreting proteases to degrade extracellular matrix $(9,23)$. Together, these different leukocytes ultimately form the immune environment and have a significant influence on the curative effects of therapies.

To combine various inflammatory biomarkers, previous studies elicited several compound systemic inflammatory indicators, including the NLR and SIRI. While NLR is a calculated inflammatory indicator of neutrophils and lymphocytes, SIRI is a parameter that integrates three types of inflammatory cells (lymphocytes, neutrophils, and monocytes), and both have been indicated to be effective prognostic indicators in several tumor models $(24,25)$. Furthermore, previous studies identified that high NLR and SIRI values were correlated with a poor prognosis, primarily in different cancer patients treated with surgery, chemotherapy, or radiotherapy $(14,15,24,26)$. To the best of our knowledge, few studies have compared the prognostic value of NLR and SIRI before radiotherapy in lung cancer. Thus, one advantage of this study was that it validated the predictive capability of pretreatment SIRI and NLR in NSCLC patients treated with chemoradiotherapy.

ROC curve analysis was also performed to evaluate the correlation between inflammatory biomarkers and prognosis. The area under the ROC curve of each inflammatory biomarker was compared, and the sensitivity and specificity of SIRI were found to be superior to those of NLR in predicting OS, while no significant cutoff values of absolute neutrophils count, lymphocytes count and monocytes count were found. In line with $\mathrm{Hu}$ et al. (24) and Valero et al. (25), we demonstrated that baseline SIRI $>0.2$ and NLR $>2.7$ could predict poor clinical outcomes for patients with locally advanced NSCLC, with the prediction ability of SIRI higher than that of NLR. The prognostic significance of SIRI remained even after adjusting for other important clinicopathological parameters, such as sex, age, smoking status, histological type, and clinical stage.

Although TNM staging system has been widely used and can predict NSCLC overall prognosis effectively, there is considerable heterogeneity between different staging groups. A precise and measurable variable that could serve as a risk indicator for survival for NSCLC patients with the same TNM stage still needs to be found. The risk stratification efficacy of SIRI supplemented by TNM stage was explored, and SIRI was demonstrated to be an effective risk stratification factor in the different stage III subgroups. Moreover, IIIB stage NSCLC patients with low SIRI $(\leq 2.0)$ were found to have better survival than IIIA stage NSCLC patients with high SIRI (>2.0), illustrating the importance of low SIRI in better survival of locally-advanced NSCLC patients. In order to obtain low SIRI, additional adjustments of clinical treatment regimens should be performed in stage III NSCLC patients.

Lymphocytes are known to be the most radiosensitive cells in the hematopoietic system; the lethal dose required to reduce the percentage of surviving lymphocytes $90 \%$ is 3 Gy (27). The degree of radiotherapy-induced lymphopenia is related to prognosis in breast cancer, rectal cancer, glioblastoma, non-small cell lung cancer, and other cancers $(18,27,28)$. However, radiotherapy planning target delineation for stage III NSCLC patients usually includes questionable lymph nodes, which obviously affects the distribution of circulating lymphocytes (29). Enhanced protection for lymph nodes during radiotherapy and a lower radiation dose to normal lymph nodes could effectively maintain the circulating absolute lymphocyte count, which may help to reduce the NLR and SIRI value and eventually promote the survival of stage III NSCLC patients. Accordingly, to guide optimal radiotherapy target delineation in clinic, in our future study, we will further explore the relationship between the elective lymph nodal irradiation dose of the target region, circulating biomarkers including NLR and SIRI, and the prognosis of locally advanced NSCLC patients. For more clarity, we should collect the data of circulating biomarkers before and after different treatment modalities, and further verify the impact of NLR and SIRI on clinical efficacy and toxicity of patients.

However, the present study has several limitations. Firstly, post-radiotherapy PBL data were lacking for the NSCLC patients in our study, which left us unable to explore the significance of increased lymph node protection in radiotherapy target delineation for SIRI and prognosis. Secondly, our study is a retrospective analysis, meaning several factors may have potentially influenced the study results; for instance, the PBL data of all patients were collected at a single time point before initial treatment, and the sample size was also relatively small. Therefore, a large-scale, multi-institutional investigation, specifically a prospective validation study, is needed to confirm our results. Despite these limitations, our findings lay the foundation for dedicated prospective investigations to corroborate better radiation delineation.

\section{Conclusions}

In summary, our study has demonstrated that pretreatment 
peripheral blood SIRI is a simple independent predictor of OS in NSCLC. As a novel prognostic marker, the prognostic value of SIRI is superior to that of NLR. Moreover, we found that pretreatment SIRI is an appropriate risk indicator that can distinguish OS on the basis of the TNM category of stage III NSCLC patients.

\section{Acknowledgments}

Funding: Three Years Action to Accelerate the Development of Traditional Chinese Medicine Plan (ZY(2018-2020)FWTX-3004).

\section{Footnote}

Reporting Checklist: The authors have completed the REMARK reporting checklist. Available at http://dx.doi. org/10.21037/atm-20-6484

Data Sharing Statement: Available at http://dx.doi. org/10.21037/atm-20-6484

Conflicts of Interest: All authors have completed the ICMJE uniform disclosure form (available at http://dx.doi. org/10.21037/atm-20-6484). The authors have no conflicts of interest to declare.

Ethical Statement: The authors are accountable for all aspects of the work in ensuring that questions related to the accuracy or integrity of any part of the work are appropriately investigated and resolved. All procedures performed in this study involving human participants were in accordance with the Declaration of Helsinki (as revised in 2013). This retrospective study received approval from the Institutional Review Boards of Shanghai Pulmonary Hospital and Zhejiang Cancer Hospital (Approval No.19231ZL). Informed consent was obtained from each patient who participated in the study.

Open Access Statement: This is an Open Access article distributed in accordance with the Creative Commons Attribution-NonCommercial-NoDerivs 4.0 International License (CC BY-NC-ND 4.0), which permits the noncommercial replication and distribution of the article with the strict proviso that no changes or edits are made and the original work is properly cited (including links to both the formal publication through the relevant DOI and the license). See: https://creativecommons.org/licenses/by-nc-nd/4.0/.

\section{References}

1. Siegel RL, Miller KD, Jemal A. Cancer statistics, 2018. CA Cancer J Clin. 2018;68:7-30.

2. Chen VW, Ruiz BA, Hsieh MC, et al. Analysis of stage and clinical/prognostic factors for lung cancer from SEER registries: AJCC staging and collaborative stage data collection system. Cancer 2014;120 Suppl 23:3781-92.

3. Palmero R, Navarro-Martin A, Nadal E. Comment on an external validation of the 8th edition of the TNM classification for lung cancer staging in patients treated with chemoradiation. Ann Transl Med 2019;7:S101.

4. Brunelli A, Kim AW, Berger KI, et al. Physiologic evaluation of the patient with lung cancer being considered for resectional surgery: Diagnosis and management of lung cancer, 3rd ed: American College of Chest Physicians evidence-based clinical practice guidelines. Chest 2013;143:e166S-90S.

5. Myall NJ, Das M. Advances in the Treatment of Stage III Non-Small Cell Lung Cancer. Clin Chest Med 2020;41:211-22.

6. Ettinger DS, Aisner DL, Wood DE, et al. NCCN Guidelines Insights: Non-Small Cell Lung Cancer, Version 5.2018. J Natl Compr Canc Netw 2018;16:807-21.

7. Grivennikov SI, Greten FR, Karin M. Immunity, inflammation, and cancer. Cell 2010;140:883-99.

8. Schreiber RD, Old LJ, Smyth MJ. Cancer immunoediting: integrating immunity's roles in cancer suppression and promotion. Science 2011;331:1565-70.

9. Mantovani A, Allavena P, Sica A, et al. Cancer-related inflammation. Nature 2008;454:436-44.

10. Deng $M$, Ma X, Liang X, et al. Are pretreatment neutrophil-lymphocyte ratio and platelet-lymphocyte ratio useful in predicting the outcomes of patients with smallcell lung cancer? Oncotarget 2017;8:37200-7.

11. Pine JK, Morris E, Hutchins GG, et al. Systemic neutrophil-to-lymphocyte ratio in colorectal cancer: the relationship to patient survival, tumour biology and local lymphocytic response to tumour. Br J Cancer 2015;113:204-11.

12. Feng JF, Huang Y, Chen QX. Preoperative platelet lymphocyte ratio (PLR) is superior to neutrophil lymphocyte ratio (NLR) as a predictive factor in patients with esophageal squamous cell carcinoma. World J Surg Oncol 2014;12:58.

13. Swierczak A, Mouchemore KA, Hamilton JA, et al. Neutrophils: important contributors to tumor progression 
and metastasis. Cancer Metastasis Rev 2015;34:735-51.

14. Geng Y, Zhu D, Wu C, et al. A novel systemic inflammation response index (SIRI) for predicting postoperative survival of patients with esophageal squamous cell carcinoma. Int Immunopharmacol 2018;65:503-10.

15. Qi Q, Zhuang L, Shen Y, et al. A novel systemic inflammation response index (SIRI) for predicting the survival of patients with pancreatic cancer after chemotherapy. Cancer 2016;122:2158-67.

16. Rami-Porta R, Asamura H, Travis WD, et al. Lung cancer - major changes in the American Joint Committee on Cancer eighth edition cancer staging manual. CA Cancer J Clin 2017;67:138-55.

17. Barbetta A, Nobel TB, Sihag S, et al. Neutrophil to Lymphocyte Ratio as Predictor of Treatment Response in Esophageal Squamous Cell Cancer. Ann Thorac Surg 2018;106:864-71.

18. Zhao Q, Chen G, Ye L, et al. Treatment-duration is related to changes in peripheral lymphocyte counts during definitive radiotherapy for unresectable stage III NSCLC. Radiat Oncol 2019;14:86.

19. Scilla KA, Bentzen SM, Lam VK, et al. NeutrophilLymphocyte Ratio Is a Prognostic Marker in Patients with Locally Advanced (Stage IIIA and IIIB) Non-Small Cell Lung Cancer Treated with Combined Modality Therapy. Oncologist 2017;22:737-42.

20. Paramanathan A, Saxena A, Morris DL. A systematic review and meta-analysis on the impact of pre-operative neutrophil lymphocyte ratio on long term outcomes after curative intent resection of solid tumours. Surg Oncol 2014;23:31-9.

21. Mandaliya H, Jones M, Oldmeadow C, et al. Prognostic biomarkers in stage IV non-small cell lung cancer (NSCLC): neutrophil to lymphocyte ratio (NLR), lymphocyte to monocyte ratio (LMR), platelet to lymphocyte ratio (PLR) and advanced lung cancer inflammation index (ALI). Transl Lung Cancer Res

Cite this article as: $\mathrm{Hu} M$, Xu Q, Yang S, Han S, Zhu Y, Lin Q, Sun X, Liu H, Xu Y. Pretreatment systemic inflammation response index (SIRI) is an independent predictor of survival in unresectable stage III non-small cell lung cancer treated with chemoradiotherapy: a two-center retrospective study. Ann Transl Med 2020;8(20):1310. doi: 10.21037/atm-20-6484
2019;8:886-94.

22. Ferrara R, Lo Russo G, Signorelli D, et al. Circulating and tumor-associated neutrophil subtypes discriminate hyperprogressive disease (HPD) from conventional progression (PD) upon immune checkpoint inhibitors (ICI) in advanced non-small cell lung cancer (NSCLC) patients (pts) and in vivo models, J Clin Oncol 2020;38:9547.

23. Singhal S, Stadanlick J, Annunziata MJ, et al. Human tumor-associated monocytes/macrophages and their regulation of $\mathrm{T}$ cell responses in early-stage lung cancer. Sci Transl Med 2019;11:eaat1500.

24. Hu B, Yang XR, Xu Y, et al. Systemic immuneinflammation index predicts prognosis of patients after curative resection for hepatocellular carcinoma. Clin Cancer Res 2014;20:6212-22.

25. Valero C, Zanoni DK, McGill MR, et al. Pretreatment peripheral blood leukocytes are independent predictors of survival in oral cavity cancer. Cancer 2020;126:994-1003.

26. Wang J, Liu Y, Mi X, et al. The prognostic value of prognostic nutritional index (PNI) and neutrophil to lymphocyte ratio (NLR) for advanced non-small cell lung cancer treated with platinum-based chemotherapeutics. Ann Palliat Med 2020;9:967-78.

27. Venkatesulu BP, Mallick S, Lin SH, et al. A systematic review of the influence of radiation-induced lymphopenia on survival outcomes in solid tumors. Crit Rev Oncol Hematol 2018;123:42-51.

28. Wang Y, Deng W, Li N, et al. Combining Immunotherapy and Radiotherapy for Cancer Treatment: Current Challenges and Future Directions. Front Pharmacol 2018;9:185.

29. Zeng X, Liu G, Pan Y, et al. Development and validation of immune inflammation-based index for predicting the clinical outcome in patients with nasopharyngeal carcinoma. J Cell Mol Med 2020;24:8326-49.

(English Language Editor: J. Reynolds) 